\title{
Secondary small cell vaginal cancer after operative therapy for endometrial cancer
}

\author{
Ikuji Shimazaki ${ }^{1}$, Yasunori Hashiguchi ${ }^{1}$, Makoto Yamauchi ${ }^{1}$, Mari Kasai ${ }^{1}$, Takeshi Fukuda ${ }^{1}$, Tomoyuki \\ Ichimura $^{1}$, Tomoyo Yasui $^{1}$, Toshiyuki Sumi ${ }^{1}$ \\ ${ }^{1}$ Department of Obstetrics and Gynecology, Osaka City University Graduate School of Medicine, Japan
}

\begin{abstract}
Summary
Background: Gynecologic neuroendocrine carcinoma is rare. There is no case report of secondary small cell vaginal cancer after operative therapy for endometrial cancer. Case: A 51 year-old patient was treated by operative therapy for endometrial cancer stage IA (pT1aN0M0). After 9 months' post-treatment, the patient visited the hospital with abnormal bleeding, and a necrotic lesion in the left upper third of the posterior vaginal wall was detected near the vaginal cut edge. Biopsy of this tumor revealed neuroendocrine carcinoma, the patient was diagnosed with small cell carcinoma of the vagina stage I (pT1NXM0) by following operative resection. The patient received chemotherapy with irinotecan and cisplatin. After eleven months of follow-up, the patient remains alive without recurrence. Conclusion: In case of small cell vaginal cancer, chemotherapy regimen with irinotecan and cisplatin should be considered as a therapeutic option.
\end{abstract}

Key words: Secondary vaginal cancer; Small cell carcinoma; Endometrial cancer.

\section{Introduction}

Neuroendocrine carcinoma of the genital tract of the female is rare, and most common sites were the ovary and uterine cervix in previous reports [1]. Primary neuroendocrine vaginal cancer is very rare, and approximately 40 cases have been reported [1-6]. There is no reported case of secondary neuroendocrine vaginal cancer. We present a rare case of secondary small cell vaginal cancer after operative therapy for endometrial cancer.

\section{Case Report}

The patient was a 51 year-old woman without past history of interest. The patient was treated by operation including hysterectomy, salpingo-oophorectomy, and pelvic lymphadenectomy for an endometrial cancer IA (T1aN0M0). The pathological examination revealed an endometrioid carcinoma, grade2 (Figure 1). The ascites showed an abnormality cytology. There was myometrial invasion $<1 / 2$ by the tumor cells. There was no abnormal findings in cervix, adnexa, or lymph node. No additional treatment was performed. After 9 months' post-treatment, the patient visited the hospital with abnormal bleeding. The pelvic examination showed a necrotic lesion in the posterior vaginal wall near the vaginal cut edge, suggesting a vaginal neoplasm. The cytological examination of vaginal wall showed atypical cells. Biopsy of this tumor revealed neuroendocrine carcinoma. Ultrasonography showed no specific findings. MRI examination of the abdomen showed no pelvic tumor or ascites. CT examination of the chest and abdomen de- tected no abnormal findings including metastasis. Laboratory tests showed no abnormal findings. Tumor marker was as followed: Neuron-specific enolase (NSE): $9.7 \mathrm{ng} / \mathrm{m}$; CA125: $9 \mathrm{U} / \mathrm{mL}$; CA19-9: $13 \mathrm{U} / \mathrm{mL}$; SCC: $0.8 \mathrm{ng} / \mathrm{mL}$; STN: $39.5 \mathrm{U} / \mathrm{mL}$. Therefore, vaginal cancer was considered the most likely diagnosis initially.

The patient underwent an operative resection. Macroscopically, no abnormal findings were detected in the peritoneal cavity. There was a necrotic lesion $(<5 \mathrm{~mm})$ in the left upper third of the posterior vaginal wall was detected near the vaginal cut edge (Figure 2). At this time, we performed resection of vaginal wall including vaginal cut edge by transabdominal and transvaginal procedure.

The pathological examination revealed the densely cellular tumor which was composed of epithelial cells with scanty cytoplasm and small hyperchromatic nuclei which were spindle to oval shaped. The tumor cells revealed strong positivity by immunostaining test for chromogranin A and CD56, although synaptophysin, p40 and cytokeratin 5/6 (CK5/6) was negative (Figure 3). Finally, we confirmed the diagnosis of small cell vaginal cancer stage I (pT1NXM0).

After operative treatment, the patient received six cycles of regimen combining irinotecan $\left(60 \mathrm{mg} / \mathrm{m}^{2}\right.$, Day $\left.1,8,15\right)$, and cisplatin $\left(60 \mathrm{mg} / \mathrm{m}^{2}\right.$, Day 1) administered. After eleven months of follow-up, the patient remains alive without recurrence.
Eur. J. Gynaecol. Oncol. - ISSN: 0392-2936 XLI, n. 5, 2020

doi: $10.31083 /$ j.ejgo.2020.05.5429
This is an open access article under the CC BY-NC 4.0 license (https://creativecommons.org/licenses/by-nc/4.0/). 


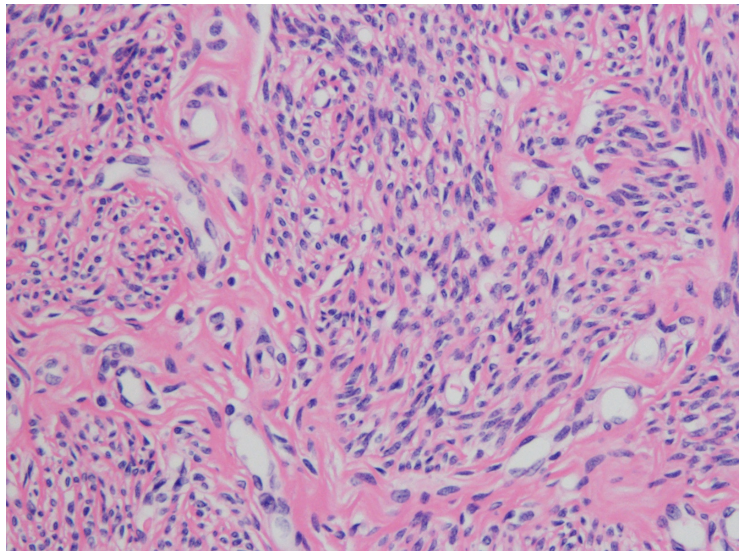

Figure 1. - The histopathological findings of previous endometrial cancer (Hematoxylin and eosin).

\section{Discussion}

Small cell carcinoma is one of types of neuroendocrine cancers. Most common site is the lungs, accounting for $20 \%$ of lung cancers [1-6]. The small cell carcinoma is rare and accounts for $2 \%$ of malignancies diagnosed in the genital tract of female. Most common sites were the ovary and uterine cervix in previous reports [1-6]. It resembles small cell lung cancer. Small cell vaginal cancer is very rare, and there are approximately 40 reported cases previously [1-6]. There is no reported case of secondary small cell vaginal cancer after hysterectomy including an operative therapy for endometrial cancer. Therefore, our case is the first reported case.

Small cell vaginal cancer reveals histological finding similar to that of small cell lung cancer. Previous reports revealed that immunohistochemistry was positive frequently for cytokeratin, NSE, synaptophysin, and chromogranin A [1]. In our case, the pathological examination revealed the densely cellular tumor which was composed of epithelial

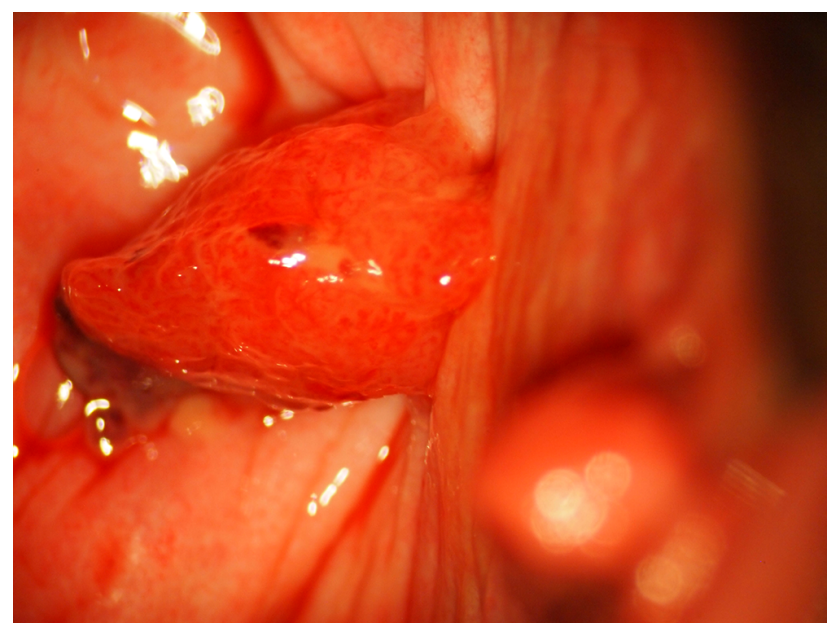

Figure 2. - A necrotic lesion in the left upper third of the posterior vaginal wall was detected near the vaginal cut edge. cells with scanty cytoplasm and small hyperchromatic nuclei which were spindle to oval shaped. The immunohistochemistry showed positive tumor for CD56 and chromogranin A, although synaptophysin, p40, and CK5/6 was negative. These features were different from the tumor cells of previous endometrial cancer. Therefore, the pathological examination revealed a small cell carcinoma, and we confirmed the diagnosis of vaginal cancer.

Small cell vaginal cancer is found at advanced stage frequently and its prognosis is miserable. Moreover, stage I patients develop progression and die within a few years in most cases, and there are only few reported cases of longterm survival [6]. There is no established treatment, since this disease is rare, and treatments have frequently resulted in miserable outcomes [1-6]. According to the previous reports, surgical resection, chemotherapy, radiation therapy, or a combination therapy is selected [6]. Local treatment is related with poor prognosis, requiring combination of therapies [3]. Oliveira R et al. reported that primary treatment is chemoradiotherapy or operative treatment followed by adjuvant chemotherapy or radiation therapy. Limited lesion in the upper third of the vagina should be resected by hysterectomy, resection of the vagina, and pelvic lymphadenectomy [3]. On the other hand, Tamura $\mathrm{R}$ reported that the patient with stage I cancer underwent radiation monotherapy, which achieved relatively long-term survival, suggesting that radiation therapy is effective therapy in small cell vaginal cancer [6]. In our case, there was a tumor in the left upper third of the posterior vaginal wall near vaginal cut edge after previous hysterectomy. There was no specific finding in the peritoneal cavity. Therefore, we performed resection of vaginal wall including vaginal cut edge by transabdominal and transvaginal procedure. Moreover, the patient developed ileus after operation. Therefore, chemotherapy was performed as adjuvant therapy to prevent postsurgical ileus by radiotherapy.

There is no established chemotherapy regimen for patients with small cell vaginal cancer. Although a combination regimen of etoposide and cisplatin is frequently administrated due to the effectiveness for pulmonary small cell carcinoma, its prognosis is miserable in reported cases $[3,6]$. On the other hand, a regimen with irinotecan and cisplatin is considered as other combination chemotherapy regimens for primary pulmonary small cell carcinoma. Five trials and two meta-analyses have investigated the regimen of etoposide and cisplatin versus irinotecan and cisplatin. Noda K reported the superiority of the irinotecan and cisplatin combination [7]. Therefore, in our case, the patient received regimen combining irinotecan and cisplatin after operation. There was no reported case performing chemotherapy combining cisplatin and irinotecan as adjuvant chemotherapy following operation for patients with small cell vaginal cancer. After eleven months of followup, the patient remains alive without recurrence. This combination chemotherapy seems to have efficacy for patients with small cell vaginal cancer. 

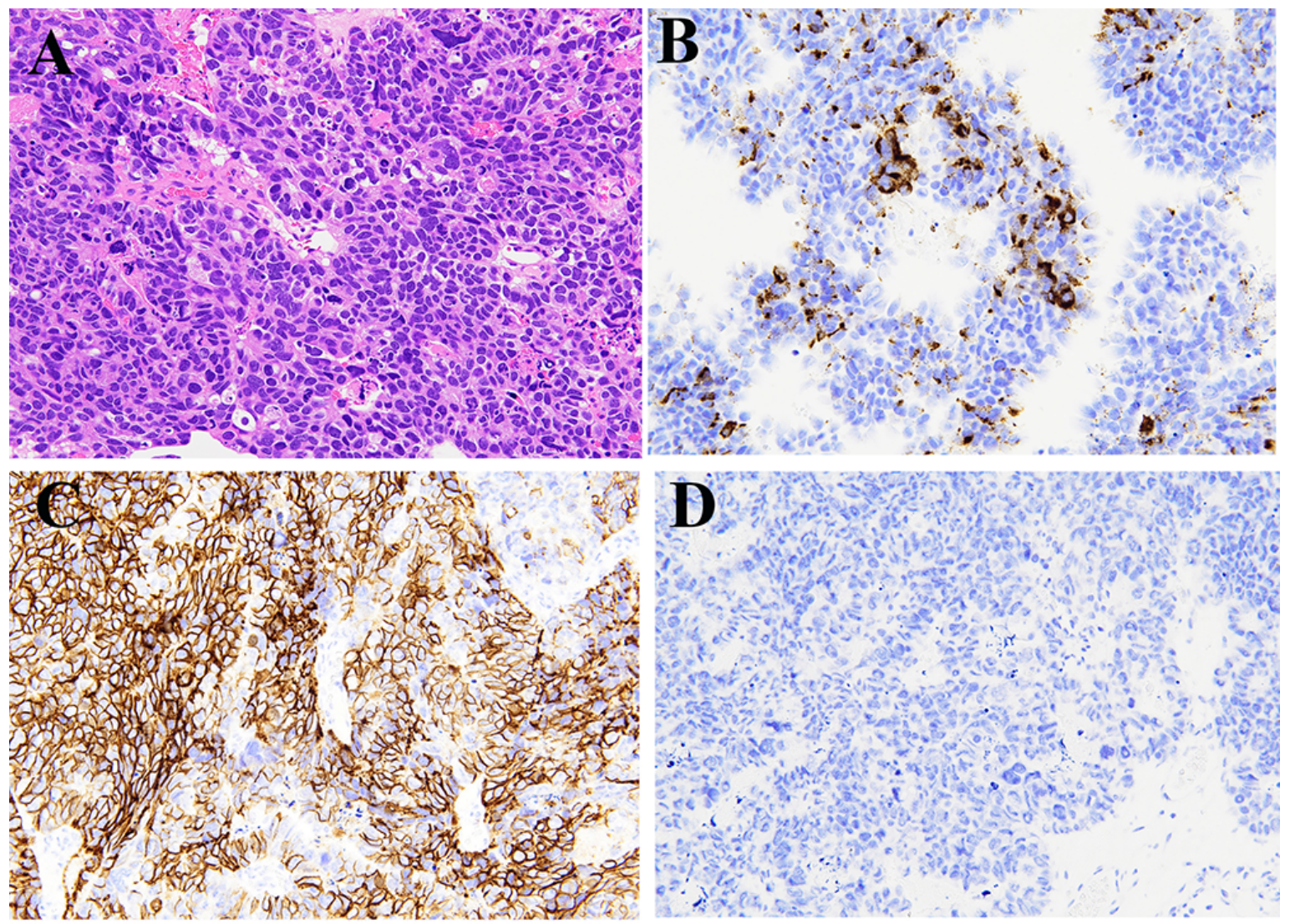

Figure 3. - (A) The histopathological findings of vaginal tumor (Hematoxylin and eosin). (B) The immunostaining was positive for chromogranin A. (C) The immunostaining was positive for CD56. (D) The immunostaining was negative for synaptophysin.

In case of small cell vaginal cancer, chemotherapy with irinotecan and cisplatin should be considered as a therapeutic option.

\section{Ethics approval and consent to participate}

Written informed consent was obtained from the patient for publication of this case report.

\section{Acknowledgments}

Thanks to all the peer reviewers and editors for their opinions and suggestions.

\section{Conflict of Interest}

The authors declare no conflict of interest.

Submitted: November 08, 2019

Accepted: May 06, 2020

Published: October 15, 2020

\section{References}

[1] Bing Z., Levine L., Lucci J.A., Hatch S.S., Eltorky M.A.: "Primary small cell neuroendocrine carcinoma of the vagina: A clinicopathologic study". Arch Pathol Lab Med, 2004, 128, 857-862.
[2] Bhalodia J.N., Kapapura D.V., Parekh M.N.: "Primary small cell neuroendocrine carcinoma of the vagina: a rare case report". Pathol Res Intt, 2011, e306921.

[3] Oliveira R., Bócoli M.C., Saldanha J.C., Murta E.F.C. and Nomelini R. S.: "Primary Small Cell Carcinoma of the Vagina". Case Reports in Obstetrics and Gynecology, 2013, 2013, 1-4.

[4] Yan W.X., Jia X.J., Chen Y.B., Zhao Z.X., Liu X.L., Yang J.Z., Wang T.J.: "Primary small cell carcinoma of the vagina with pulmonary metastasis: a case report". Eur J Gynaecol oncol, 2016, 37, 129-132.

[5] Kombathula S.H., Rapole P.S., Prem S.S. and Badhe B.: "Primary small cell carcinoma of the vagina: a rare instance of prolonged survival". Bmj Case Reports, 2019, 12, e227100.

[6] Tamura R., Yokoyama Y., Kobayashi A., Osawa Y., Shigeto T., Futagami M., et al.: "A Case of Small Cell Carcinoma of the Vagina". Rare Tumors, 2013, 5, 189-191.

[7] Noda K., Nishiwaki Y., Kawahara M., Negoro S., Sugiura T., Yokoyama A., et al.: "Irinotecan plus Cisplatin Compared with Etoposide plus Cisplatin for Extensive Small-Cell Lung Cancer", N. Engl. J. Med., 2002, 346, 85-91.

Corresponding Author:

YASUNORI HASHIGUCHI, M.D.

Department of Obstetrics and Gynecology, Osaka City University Graduate School of Medicine, 1-4-3 Asahimachi, Abeno-ku, Osaka 545-8585 (Japan) e-mail: hashiguchi@med.osaka-cu.ac.jp 